\title{
Isolation and Characterization of Extracellular Vesicle from Mesenchymal Stem Cells of the Epidural Fat of the Spine
}

\author{
Soo-Eun Sung ${ }^{1,}$, Min-Soo Seo ${ }^{1,}$, Kyung-Ku Kang ${ }^{1}$, Joo-Hee Choi ${ }^{1}$, Si-Joon Lee ${ }^{1}$, \\ Ju-Hyeon Lim ${ }^{2,3}$, Seung Yun Yang ${ }^{4}$, Seul-Ki Kim ${ }^{5}$, Gun Woo Lee ${ }^{3}$ \\ ${ }^{1}$ Department of Laboratory Animal Center, Daegu-Gyeongbuk Medical Innovation Foundation, Daegu, Korea \\ ${ }^{2}$ New Drug Development Center, Osong Medical Innovation Foundation, Cheonju, Korea \\ ${ }^{3}$ Department of Orthopaedic Surgery, Yeungnam University Medical Center, Yeungnam University College of Medicine, Daegu, Korea \\ ${ }^{4}$ Department of Biomaterials Science (BK21 Four Program), Pusan National University, Pusan, Korea \\ ${ }^{5}$ Efficacy Evaluation Team, Food Science R\&D Center, KolmarBNH Co. Ltd., Seoul, Korea
}

Study Design: An experimental study with extracellular vesicles (EVs) from mesenchymal stem cell (MSC) of the epidural fat (EF) of the spine.

Purpose: This study aims to isolate the exosomes from epidural fat-derived mesenchymal stem cells (EF-MSCs) and fully characterize the EF-MSC-EVs.

Overview of Literature: EF-MSCs were reported in 2019, and a few studies have shown the positive outcomes of using EF-MSCs to treat specific spine pathologies. However, MSCs have significant limitations for conducting basic studies or developing therapeutic agents. Although EVs are an emerging research topic, no studies have focused on EVs, especially exosomes, from EF and EF-MSCs.

Methods: In this study, we isolated the exosomes using the tangential flow filtration (TFF) system with exosome-depleted fetal bovine serum and performed the characterization tests via western blotting, reverse transcription-polymerase chain reaction, nanoparticle tracking analysis (NTA), and transmission electron microscopy.

Results: In transmission electron microscopy, the exosome had a diameter of approximately 100-200 nm and had a spherical shape, whereas in the NTA, the exosome had an average diameter of $142.8 \mathrm{~nm}$ with a concentration of $1.27 \times 10^{10}$ particles $/ \mathrm{mL}$. The flow cytometry analysis showed the expression of CD63 and CD81. The western blotting analysis showed the positive markers.

Conclusions: These findings showed that isolating the exosomes via TFF resulted in high-quality EF-MSC exosome yield. Further studies with exosomes from EF-MSC are needed to evaluate the function and role of the EF tissue.

Keywords: Epidural fat; Mesenchymal stem cells; Extracellular vesicles; Exosomes; Isolation

Received Apr 7, 2021; Revised May 3, 2021; Accepted May 11, 2021

Corresponding author: Gun Woo Lee

Department of Orthopaedic Surgery, Yeungnam University Hospital, Yeungnam University College of Medicine, 170 Hyeonchung-ro, Nam-gu, Daegu 42415, Korea

Tel: +82-53-620-3642, Fax: +82-53-628-4020, E-mail: gwlee1871@gmail.com, gwlee1871@ynu.ac.kr

*These authors contributed equally to this work, as the first author. 


\section{Introduction}

Epidural fat (EF) is generally located in the epidural space of the spine, but no detailed study has evaluated the specific role, function, and clinical relevance of the EF tissue itself. To date, only a basic study has indicated that EF contains mesenchymal stem cells (MSCs) [1,2]. A few studies have shown that MSCs derived from EF can produce an affirmative effect for specific pathologies in the spine, such as spinal cord injury and post-lumbar surgery syndrome $[2,3]$. However, spine physicians are illinformed on $\mathrm{EF}$.

Many studies have demonstrated that MSCs have significant functions in target structures or diseases [4]. However, MSCs have significant limitations for conducting further studies or developing therapeutic agents, such as ethical issues and problems of storage or handling $[5,6]$. Recently, extracellular vesicles (EVs) have been popularized as a vital source in the field of regenerative medicine [7-9]. EVs can be obtained from almost all cells, including MSCs and human fluids, but some have argued that EVs from different origins may play distinct roles [9]. Moreover, considering the characteristics of MSCs, EVs obtained from specific cells may have specialized functions with respect to nearby structures of the donor tissue. However, this point of contention has not been fully addressed.

Based on a hypothesis, we conducted several experimental and clinical studies on EF. Our previous study demonstrated that EF contains MSCs [1], which may have clinical implications for spine pathologies. For this study, our aim was to report the first EVs isolated from EF-MSCs and fully characterized in a defined manner. We hypothesized that EVs from EF-MSCs would exhibit regenerative properties, which may have specialized functions in spine-related pathologies.

\section{Materials and Methods}

\section{Cell isolation and culture}

EF was obtained from consenting patients $(n=5)$ during posterior decompression surgery of the lumbar spine performed at Yeungnam University Medical Center (Table 1). The specimens were placed in sterile tubes, stored at $4^{\circ} \mathrm{C}$, and brought to the Laboratory Animal Center of the Daegu-Gyeongbuk Medical Innovation Foundation to be processed within 12 hours.

Human EF-MSCs were separated from the EF tissue. In brief, the $\mathrm{EF}$ tissues were rinsed in $70 \%$ ethanol and icecold phosphate buffered saline (PBS) and digested using $0.45 \mu \mathrm{m}$ filtered $2 \mathrm{mg} / \mathrm{mL}$ collagenase type I (17018029, Gibco; Invitrogen, Carlsbad, CA, USA) at $37^{\circ} \mathrm{C}$ for 30 minutes. The solution was passed through a $70 \mu \mathrm{m}$ strainer and centrifuged at $3,000 \times \mathrm{g}$ for 5 minutes. The pellets were suspended in a culture medium and seeded on culture dishes. After 1 day, the cells were washed with PBS, and debris, red blood cells, and so forth were discarded. EF-MSCs were maintained in low-glucose DMEM (11885092, Gibco; Invitrogen) supplemented with 10\% exosome-depleted fetal bovine serum (FBS) (Gibco, USA origin; Invitrogen), 1\% penicillin/streptomycin (Gibco; Invitrogen), $10 \mu \mathrm{g} / \mathrm{mL}$ recombinant human FGF-basic (100-18B; Peprotech, Cranbury, NJ, USA), $10 \mu \mathrm{g} / \mathrm{mL}$ recombinant human PDGF-BB (500-P47; Peprotech), and $25 \mu \mathrm{g} / \mathrm{mL}$ Plasmocin prophylactic (ant-mpp; InvivoGen, San Diego, CA, USA) to prevent mycoplasma contamination. They were cultured at $37^{\circ} \mathrm{C}$ in a $5 \% \mathrm{CO}_{2}$ incubator.

\section{Isolation of the epidural fat-derived mesenchymal stem cells exosomes}

The culture medium used to grow EF-MSC in T-175 flasks

Table 1. Demographic data

\begin{tabular}{lccclc} 
Case & Age $(\mathrm{yr})$ & Sex & Body mass index $\left(\mathrm{kg} / \mathrm{m}^{2}\right)$ & Diagnosis & Treatment \\
Case 1 & 47 & M & 26.8 & HIVD L4-5 & Open discectomy \\
Case 2 & 53 & F & 29.4 & LSS L4-5-S1 & Posterior decompression \\
Case 3 & 39 & F & 30.2 & HIVD L5-S1 & Open discectomy \\
Case 4 & 44 & M & 24.9 & HIVD L4-5 & Open discectomy \\
Case 5 & 51 & M & 27.3 & HIVD L4-5 & Open discectomy \\
\hline
\end{tabular}

M, male; F, female; HIVD, herniated intervertebral disc; LSS, lumbar spinal stenosis. 
was collected after 48 hours. The cells were $80 \%-90 \%$ confluent with a viability of $>95 \%$. The media were stored at $4^{\circ} \mathrm{C}$ and pooled together to make up a $200 \mathrm{~mL}$ volume. The collected medium was centrifuged at $300 \times \mathrm{g}$ for 10 minutes to remove cells and debris. The supernatant was filtered through a tangential flow filtration (TFF) system (Minimate TFF System; Pall Corp., Port Washington, NY, USA). The feed flow rate was approximately $2.5 \mathrm{~mL} /$ $\mathrm{min}$, and the total product volume was $6 \mathrm{~mL}$. The membrane nominal pore size was $10 \mathrm{~nm}(100 \mathrm{~K}$, Minimate TFF Capsule; Pall Corp.). The exosome protein concentration was measured using the Pierce bicinchoninic acid (BCA) Assay Kit (23225; Thermo Fisher Scientific, Rockford, IL, USA).

\section{Reverse transcription-polymerase chain reaction}

Total RNA was isolated using an RNeasy Mini Kit (74106; QIAGEN, Hilden, Germany) according to the manufacturer's protocol. The RNA concentration was determined using a NanoDrop spectrophotometer (Thermo Fisher Scientific). To synthesize cDNA, total RNA and a SuperScript III First-Strand Synthesis System (18080051; Thermo Fisher Scientific) were used. The synthesized cDNA was amplified using the primers listed in Table 2. The amplified polymerase chain reaction (PCR) products were visualized using 1.5\% (weight/volume [w/v]) agarose gel electrophoresis.

\section{Flow cytometry}

The flow cytometry analyses were performed using a Gallios flow cytometer (Beckman Coulter, Brea, CA, USA). To determine the expression of the stem cell markers, the cells were stained with antibodies, such as CD105 (MCA1557; Bio-Rad, Hercules, CA, USA), CD90 (555596;
BioLegend, San Diego, CA, USA), CD73 (344004; BioLegend), CD45 (555482; BioLegend), CD34 (343504; BioLegend), and CD14 (MCA1568, Bio-Rad). The antibodies were conjugated with fluorescein isothiocyanate (FITC) or phycoerythrin fluorescence dye. To analyze the isolated exosomes, they were incubated with $4 \% \mathrm{w} / \mathrm{v}$ aldehyde/ sulfate-latex beads (A37304; Thermo Fisher Scientific) for 15 minutes at room temperature. Then, $1 \mathrm{~mL}$ PBS (supplemented with $0.1 \%$ bovine serum albumin) was added to the exosome-bead mixture. The samples were incubated overnight on rotation. Bead-coupled exosomes were pelleted by centrifugation at $2,000 \times \mathrm{g}$ for 10 minutes and washed with $500 \mu \mathrm{L}$ PBS. The pellets were re-suspended in $50 \mu \mathrm{L}$ PBS containing the antibodies, CD9 (NBP1-28364; Novus Biologicals, Centennial, CO, USA), and CD81 (NBP1-44859; Novus Biologicals) for 1 hour at $4^{\circ} \mathrm{C}$. All the antibodies were conjugated with FITC fluorescence dye. The samples were washed using $500 \mu \mathrm{L}$ PBS and centrifuged at 2,000 $\times \mathrm{g}$ for 10 minutes. The pellets were resuspended in PBS. The gating of exosome-decorated $4 \mu \mathrm{m}$ diameter beads was analyzed using a Gallios flow cytometer. The flow cytometry analyses were performed using the Kaluza software (Beckman Coulter).

\section{Western blotting}

To identify the exosome markers, the exosomes were extracted with RIPA lysis buffer (89900; Thermo Fisher Scientific) containing a protease inhibitor (87786; Thermo Fisher Scientific). The exosome lysates were mixed with LDS Sample Buffer (B0007; Thermo Fisher Scientific) and boiled at $95^{\circ} \mathrm{C}$ for 5 minutes. Then, $25 \mu \mathrm{g}$ of protein was electrophoresed on 4\%-12\% Bis-Tris Plus gels (NP0335; Thermo Fisher Scientific) with MOPS SDS running buffer (B0001, NuPAGE; Thermo Fisher Scientific). The polyvinylidene fluoride membranes (LC2002; Thermo Fisher

Table 2. Nucleotide sequence of primers used for reverse transcription-polymerase chain reaction

\begin{tabular}{lll} 
Gene & Forward sequence $\left(5^{\prime}-3^{\prime}\right)$ & Reverse sequence $\left(5^{\prime}-3^{\prime}\right)$ \\
OCT4 & CTTCAGGAGATATGCAAAGCA & ACACTCGGACCACATCCTTC \\
Sox2 & TTGCAATATTITCAAGGAGA & CAAGACCACAGAGATGGTTCG \\
c-Myc & GCGACTCTGAGGAGGACAA & TGCGTAGTGTGCTGATGTG \\
KIf4 & AGGCACTACCGTAAACACACG & CGGGAAGACAGTGTGAAAGT \\
\hline GAPDH & CGCTGAGTACGTCGTGGAGT & GGAGGCATTGCTGATGATCT \\
\hline
\end{tabular}

GAPDH, glyceraldehyde-3-phosphate dehydrogenase. 
Scientific) were blocked with 5\% skimmed milk for 1 hour at room temperature. The cells were then incubated overnight with primary antibodies against glyceraldehyde3-phosphate dehydrogenase (GAPDH, NB100-56875; Novus Biologicals), calnexin (NB100-1965; Novus Biologicals), CD9 (NBP1-28363; Novus Biologicals), CD81 (NBP1-44861; Novus Biologicals), flotillin 2 (NBP130881; Novus Biologicals), and TSG101 (NB200-112; Novus Biologicals) at $4^{\circ} \mathrm{C}$. The cells were then incubated with the horseradish peroxidase-conjugated secondary antibodies, anti-mouse immunoglobulin G (IgG, 31430; Thermo Fisher Scientific), and anti-rabbit IgG (31460; Thermo Fisher Scientific). Western blot development was performed using ECL (enhanced chemiluminescence) solution (2332638, EzWest Lumi plus; ATTO, Tokyo, Japan).

\section{Transmission electron microscopy}

Freshly isolated exosomes from hEpi AD-MSCs were re-suspended in cold distilled water. The exosome suspensions were loaded on formvar carbon-coated grids (01800-F; Ted Pella Inc., Redding, CA, USA) and fixed in $2 \%$ paraformaldehyde for 10 minutes. Then, the solutions were removed and the samples were dried. The grids were observed using bioTEM (HT7700; Hitachi, Tokyo, Japan).

\section{Nanoparticle tracking analysis}

To determine the size distribution and average size of the exosomes, nanoparticle tracking analysis (NTA) was performed using PMX-120 ZetaView (Particle Metrix, Meerbusch, Germany). Briefly, the exosomes were diluted in PBS to a final volume of $1 \mathrm{~mL}$. The ideal exosome concentrations were found via pretesting. The exosomes could be observed through the microscope. Finally, the NTA system was flushed with a sufficient amount of PBS $[10,11]$.

\section{Serum biochemistry}

Albumin concentration was measured using a TBA120FR analyzer (Toshiba, Tokyo, Japan) with the Wako ALB reagent (FUJIFILM, Tokyo, Japan).

\section{Ethical considerations}

This study was approved by the Institutional Review
Board and Ethical Committee of Yeungnam University Medical Center (IRB approval no., 2017-07-032).

\section{Results}

\section{Characteristics of the epidural fat-derived mesenchy- mal stem cells}

EF-MSCs were isolated from human epidural adipose tissue. The isolated EF-MSCs exhibited typical MSC characteristics. The cells were long, thin, and spindle-shaped, similar to general fibroblasts (Fig. 1A). MSCs are known to express stemness markers, such as Oct4, Sox2, C-myc, and Klf4. The isolated hEpi AD-MSCs also expressed all the stemness markers (Fig. 1B). The isolated cells were analyzed for specific cell surface markers using flow cytometry. The cells were positive for the MSC-associated markers, CD73, CD90, and CD105. On the contrary, they were negative for the hematopoietic markers, CD14, CD34, and CD45 (Fig. 1C-H). The cells were passaged up to 12 times for the proliferation of stem cells and isolation of EVs. Furthermore, results showed that the isolated hEpi AD-MSCs had typical characteristics of MSCs.

\section{Epidural fat-derived mesenchymal stem cells exo- somes isolated using the tangential flow filtration system}

The exosomes were observed under a transmission electron microscope (TEM). The exosome was approximately 100-200 nm in diameter and spherical in shape. Moreover, since the exosome membrane was composed of a lipid bilayer, dark and thick exosome membranes were observed under TEM (Fig. 2A). A NTA was used to determine the size distribution and particle numbers in a milliliter of solution. Of the isolated EF-MSC-derived exosomes, $99.1 \%$ had an average diameter of $142.8 \mathrm{~nm}$, and the exosomes had a concentration of $1.27 \times 10^{10}$ particles $/ \mathrm{mL}$ (Fig. $2 \mathrm{~B}$ ). Hence, the total number of isolated exosome particles was $7.62 \times 10^{10}$ particles in $200 \mathrm{~mL}$ of cell culture medium. Moreover, aldehyde/latex beads with a diameter of approximately $4 \mu \mathrm{m}$ were used with exosomes to aid detection by a flow cytometry analyzer. The bead-binding exosomes were analyzed via flow cytometry, which detected approximately $63.4 \%$ and $98.4 \%$ of the expressed CD63 and CD81, respectively (Fig. 2C, D). The western blotting analysis showed that the exosome 


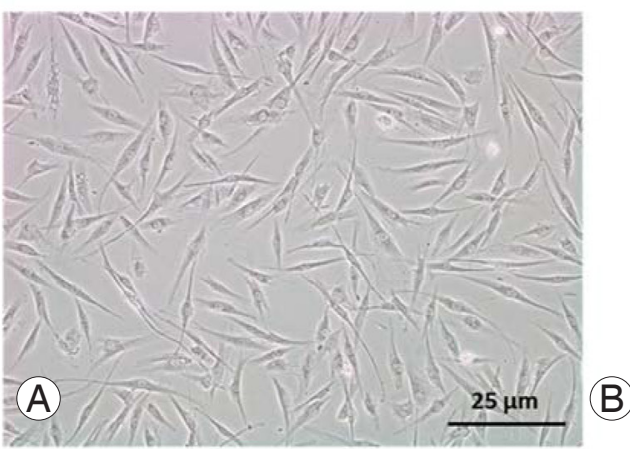

$\mathrm{CD73}$
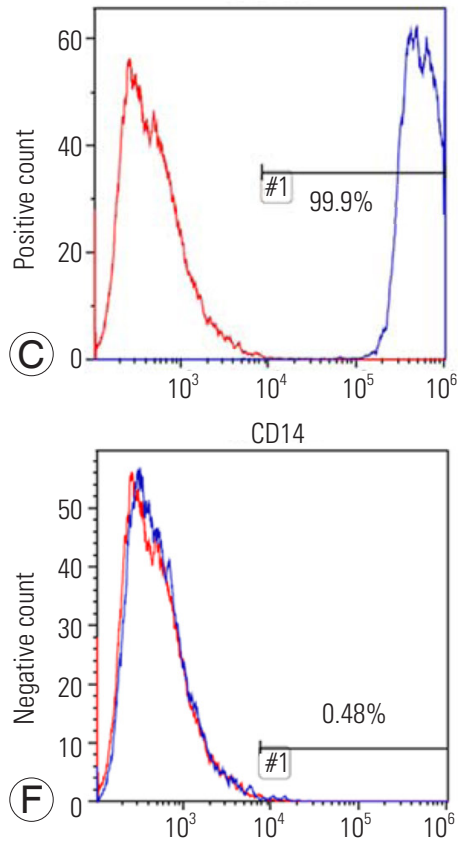

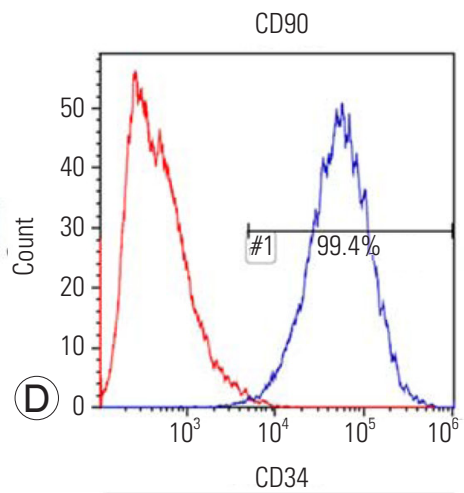

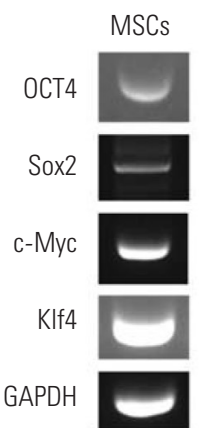

Fig. 1. Characteristics of human epidural fatderived mesenchymal stem cells (EF-MSCs). (A) EF-MSCs were observed under a light microscope (magnification, $\times 100$ ). (B) Reverse transcription-polymerase chain reaction showed that EF-MSCs expressed stem cells markers (OCT4, Sox2, C-Myc, and KIf4). (C-H) EF-MSCs were analyzed using flow cytometry, and were positive (CD73, CD90, and CD105) and negative (CD14, CD34, and CD45) for different markers. GAPDH, glyceraldehyde-3-phosphate dehydrogenase.
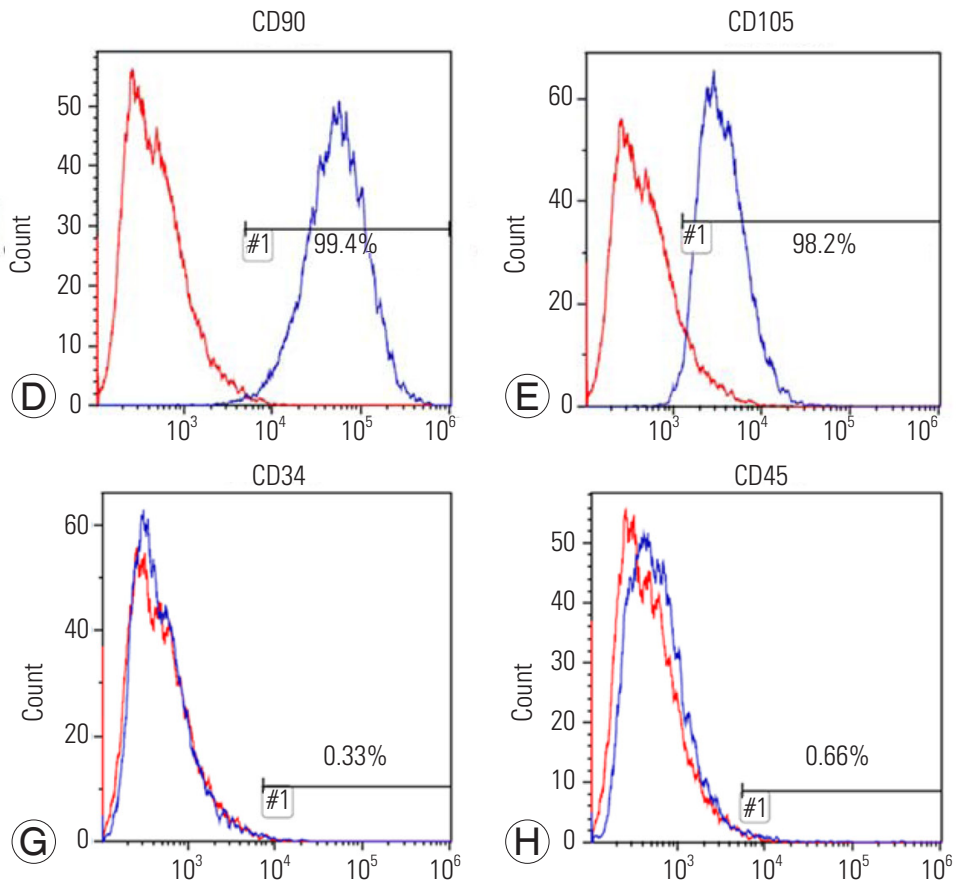

markers CD9 and CD81, exosome fusion protein flotillin 2, multi-vesicular bodies (MVB) biogenesis proteins (TSG101), and intracellular cytosolic marker calnexin were expressed (Fig. 2E). The total protein concentration was $4,050 \mu \mathrm{g} / \mathrm{mL}$, based on a BCA assay. These findings showed that isolating the exosomes via TFF resulted in high-quality EF-MSC exosome yield.

\section{Discussion}

Our study has several strength points regarding the evaluation of the exosome from EF-MSC. First, previous studies on EVs have focused on contamination, especially with albumin. To harvest EF-MSCs that secrete EVs, the cell culture medium should be serum-free or xeno-free to inhibit contamination. However, such media contain many albumins and other proteins. Albumin was analyzed using serum biochemistry instruments. Commonly used media, such as FBS, culture medium containing exosome-depleted FBS, and serum-free commercial media, contained $0.16,0.03$, and $0.13 \mathrm{~g} / \mathrm{dL}$ albumin, respectively. Hence, cell culture medium containing exosome-depleted FBS was used to enhance the purity of isolated EVs. To prove this, a NTA was used to determine the size distribution and particle numbers in a milliliter of solution. Of the isolated EF-MSC-derived exosomes, 99.1\% had an average diameter of $142.8 \mathrm{~nm}$, and the exosomes had a concentration of $1.27 \times 10^{10}$ particles $/ \mathrm{mL}$. The total number of isolated exosome particles was $7.62 \times 10^{10}$ particles in $200 \mathrm{~mL}$ of cell culture medium (Fig. 2). These NTA findings showed that isolating the exosomes via TFF with the culture medium resulted in high-quality exosome yield. Third, we 
(A)

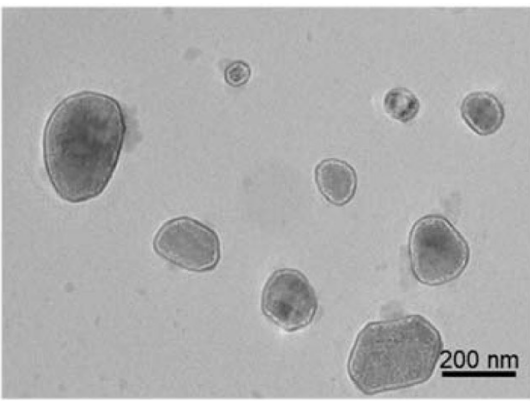

(B)

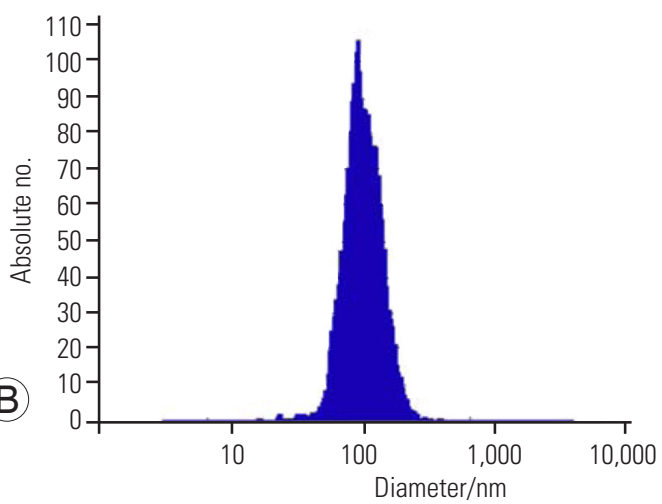

CD81
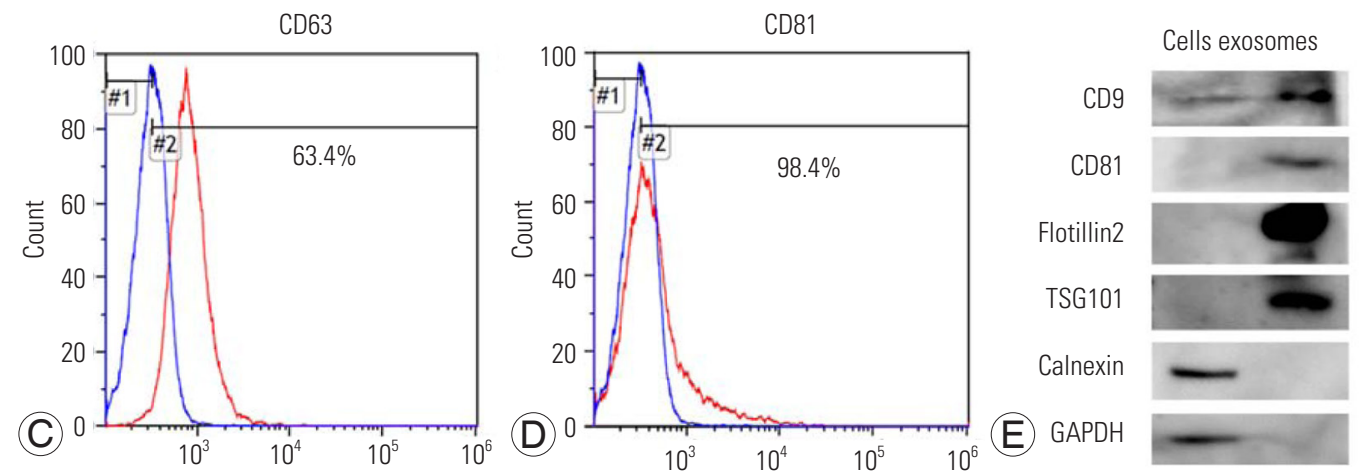

Fig. 2. Human epidural fat-derived mesenchymal stem cell (EF-MSC) exosomes isolated using tangential flow filtration system. (A) Representative image of exosomes from EF-MSCs visualized using transmission electron microscopy, size bar=200 nm. (B) Nanoparticle tracking analysis profile of human EF-MSCs exosomes showing size distribution. Isolated particles had an average size of approximately $142.8 \mathrm{~nm}$ and the concentration of particles was $1.27 \times 10^{10}$ particles $/ \mathrm{mL}$. (C, D) Flow cytometry analysis of exosomes isolated from human EF-MSCs. Exosomes were labeled using the tetraspanins, CD63 (upper panel) and CD81 (below panel), which exhibited $63.4 \%$ and $98.4 \%$ expression, respectively. (E) Western blots stained against tetraspanins (CD9 and CD81), exosome fusion protein (Flotillin2), and MVB biogenesis protein (TSG101). The positive expression of the cell markers, calnexin and glyceraldehyde3-phosphate dehydrogenase (GAPDH), in human EF-MSCs was detected using western blotting.

performed some well-structured methods for exosome characterization. Exosomes derived from MVBs express several biomarkers, such as tetraspanins, fusion proteins, and MVB biogenesis markers. In particular, tetraspanins, such as CD9, CD63, and CD81, are expressed outside the exosome membrane. Therefore, we detected the tetraspanins, CD63 and CD81, via flow cytometry. Flow cytometry analyses are generally performed for cells. However, exosomes are much smaller than common cells. Hence, aldehyde/latex beads with a diameter of approximately $4 \mu \mathrm{m}$ were used with exosomes to aid detection using a flow cytometry analyzer. Bead-binding exosomes were analyzed via flow cytometry, which detected approximately $63.4 \%$ and $98.4 \%$ of the expressed CD63 and CD81, respectively (Fig. 2). Western blotting analysis was also performed to detect the exosome markers, tetraspanins CD9 and CD81, exosome fusion protein flotillin 2, and MVB biogenesis proteins (TSG101). The intracellular cytosolic marker, calnexin, was expressed in cell lysates. Interestingly, the isolated EF-MSC exosomes did not express GAPDH (Fig. 2). Moreover, the total protein concentration was 4,050 $\mu \mathrm{g} / \mathrm{mL}$, based on a BCA assay. These findings showed that isolating the exosomes via TFF resulted in high-quality EF-MSC exosome yield.

To date, few articles have focused on EF itself or the function of the EF tissue. Some papers have reported histopathological findings related to EF $[12,13]$. Topographically, EF is arranged around the dural sac and extends along the entire spine in the fetus. However, the arrangement differs in adults; it is discontinuous, metameric, and mainly in the posterior part of the epidural space [13]. Importantly, EF is covered by a thin membrane of connective tissue in the form of a loose, areolar, and thin layer. Under this layer, EF is freely located. Feeding vessels from a specific connective tissue, in the form of pedicle-like attachments, are located beneath the posterior surface of the 
EF. Microscopically, EF is a homogenous tissue; it scarcely contains connective tissue and oriented slits and is semifluidic in nature. It consists of adipocytes that are uniform in size and shape, with features that significantly differ from those of subcutaneous fat [12-16]. Based on the histopathological findings of previous studies, we could presume the mechanical roles of the EF. Firstly, EF is not just a space-filling tissue in the epidural space. Since EF has a specific tissue arrangement and composition, contrary to subcutaneous fat tissue, it may have its own function or role at the site. Secondly, EF consists of minimal connective tissue with oriented slits and homogenous arrangement of cells. These features of the EF may facilitate the sliding of the dura mater against the posterior structures of the spine, such as the ligamentum flavum and bony structures, and may function to protect the neural structures, especially during spine movement.

Although some studies have suggested the histopathological features of the EF, subsequent studies have rarely reported a better definition of its function. Spine physicians rarely pay attention to EF, and it is mostly linked to epidural fibrosis formation after spinal surgery [17-26] and clinically uncharacterized back pain [24,27,28]. Recently, novel studies with different views on EF have focused on its regenerative or immunomodulatory function, especially with respect to MSCs derived from EF. In 2019, two studies demonstrated that EF contains MSCs and EFMSCs may play various significant roles with respect to nearby structures $[1,2]$. Specifically, MSCs and progenitor cells derived from EF showed collagen contraction capacity and a fibroblast-like phenomenon [2]. Based on an in vivo analysis of MSCs and progenitor cells from EF, AlJezani et al. [2] suggested that the cell population may contribute to the neural structures and EF cells may play a biological role within the local environment in addition to enacting mechanical roles. However, several difficulties have been observed in conducting basic studies with EF-MSCs. To date, EVs have been considered as novel source materials for regenerative studies and clinical relevance $[3,7-9,29]$. EVs exhibit several advantages for such research, and one of their advantages over MSCs is that they do not consume their donor tissues or cells. Moreover, EVs are acellular nano-sized vesicles with nucleic acids (mRNA, miRNA, etc.) and functional proteins and can mediate cell-cell communication and cell signaling. Recent studies have also demonstrated that exosomes, a subgroup of EVs, play significant roles in a variety of pro- cesses, such as angiogenesis, immunomodulation, antiinflammatory activity, and homeostasis regulation [9]. Moreover, these potential roles of exosomes are comparable to those of MSCs. Since certain MSCs affect nearby tissues or cells of donors, exosomes from EF-MSCs can also positively affect the structures surrounding the EF tissue, such as neural structures, disks, and bony structures. Moreover, based on the advantages of EVs over MSCs for basic research, exosomes should be evaluated to determine their roles. From this perspective, the present study is the first study wherein exosomes were isolated from EFMSCs. Moreover, owing to those positive points of EVs from EF or EF-MSC, they may have a clinical significance for the treatment of spine diseases, such as degenerative spine disease, spinal cord injury, and others. Further studies with EVs from EF itself or EF-MSC are needed to better understand its meaning and clinical significance.

However, this study has some limitations. Firstly, we have described the exosome features of EF-MSCs without comparisons to other exosomes. To date, most MSC studies have used subcutaneous tissue-derived MSCs as donor tissues. Hence, a comparison of the exosomes from EFMSCs and those derived from subcutaneous tissues may be instructive. Considering this, we intend to conduct a comparative study of these two types of MSC-derived exosomes. Secondly, the unique features of exosomes from EF-MSCs were experimentally investigated. The present study is fundamental for further experimental and clinical investigations on EF-MSC-derived exosomes. Therefore, further studies are needed to define their significance. Despite these limitations, this study has significant strengths. This is the first study to identify exosomes from EF-MSCs, which can be vital tools for treating specific pathologies and may especially have a critical impact on spine pathologies. Therefore, this study can be a cornerstone for further research using exosomes from EF-MSCs and regenerative treatments for spine pathologies.

\section{Conclusions}

To the best of our knowledge, this is the first study wherein exosomes were isolated from EF-MSCs. A TFF system was used for cell isolation, and characterizations were performed via western blotting, reverse transcription-PCR, BCA, NTA, and TEM. All of the features of the samples corresponded to those of the exosomes from EF-MSCs with high purity and yield. Further experimental and 
clinical studies with exosomes from EF-MSCs are needed to determine the function and role of the EF tissue.

\section{Conflict of Interest}

No potential conflict of interest relevant to this article was reported.

\section{Funding}

This study was supported by the Bio \& Medical Technology Development Program of the National Research Foundation (NRF), funded by the Ministry of Science \& ICT (2019M3E5D1A02068105). The funders had no role in study design, data collection and analysis, decision to publish, or preparation of the manuscript.

\section{Author Contributions}

Conception and design: Lee GW, Seo MS; data acquisition: Sung SE, Seo MS, Kang KK, Choi JH, Lee SJ, Lim JH, Yang SY, Kim SK, Lee GW; analysis of data: Seo MS, Lee GW; drafting of the manuscript: Sung SE, Lee GW; critical revision: Lee GW; obtaining funding: Lee GW; administrative support: Lee GW; and supervision: Seo MS, Lee GW.

\section{References}

1. Lee GW, Seo MS, Kang KK, Oh SK. Epidural fat-derived mesenchymal stem cell: first report of epidural fat-derived mesenchymal stem cell. Asian Spine J 2019;13:361-7.

2. Al-Jezani N, Cho R, Masson AO, Lenehan B, Krawetz $\mathrm{R}$, Lyons FG. Isolation and characterization of an adult stem cell population from human epidural fat. Stem Cells Int 2019;2019:2175273.

3. Shah S, Mudigonda S, Mitha AP, Salo P, Krawetz RJ. Epidural fat mesenchymal stem cells: important microenvironmental regulators in health, disease, and regeneration: do EF-MSCs play a role in dural homeostasis/maintenance? Bioessays 2021;43:e2000215.

4. Baer PC. Adipose-derived stem cells and their potential to differentiate into the epithelial lineage. Stem Cells Dev 2011;20:1805-16.

5. Cristofalo VJ, Allen RG, Pignolo RJ, Martin BG, Beck JC. Relationship between donor age and the replica- tive lifespan of human cells in culture: a reevaluation. Proc Natl Acad Sci U S A 1998;95:10614-9.

6. Oberringer $M$, Bubel $M$, Jennewein $M$, et al. The role of adipose-derived stem cells in a self-organizing 3D model with regard to human soft tissue healing. Mol Cell Biochem 2018;445:195-210.

7. Kim GB, Shon OJ, Seo MS, Choi Y, Park WT, Lee GW. Mesenchymal stem cell-derived exosomes and their therapeutic potential for osteoarthritis. Biology (Basel) 2021;10:285.

8. Qing L, Chen H, Tang J, Jia X. Exosomes and their MicroRNA cargo: new players in peripheral nerve regeneration. Neurorehabil Neural Repair 2018;32:76576.

9. Lai RC, Yeo RW, Lim SK. Mesenchymal stem cell exosomes. Semin Cell Dev Biol 2015;40:82-8.

10. Lujan H, Griffin WC, Taube JH, Sayes CM. Synthesis and characterization of nanometer-sized liposomes for encapsulation and microRNA transfer to breast cancer cells. Int J Nanomedicine 2019;14:5159-73.

11. Bachurski D, Schuldner M, Nguyen PH, et al. Extracellular vesicle measurements with nanoparticle tracking analysis: an accuracy and repeatability comparison between NanoSight NS300 and ZetaView. J Extracell Vesicles 2019;8:1596016.

12. Reina MA, Franco CD, Lopez A, De Andres JA, van Zundert A. Clinical implications of epidural fat in the spinal canal: a scanning electron microscopic study. Acta Anaesthesiol Belg 2009;60:7-17.

13. Beaujeux R, Wolfram-Gabel R, Kehrli P, et al. Posterior lumbar epidural fat as a functional structure?: histologic specificities. Spine (Phila Pa 1976) 1997;22:1264-8.

14. Reina MA, Pulido P, Castedo J, Villanueva MC, Lopez A, Sola RG. Characteristics and distribution of normal human epidural fat. Rev Esp Anestesiol Reanim 2006;53:363-72.

15. Reina MA, Pulido P, Castedo J, et al. Epidural fat in various diseases: contribution of magnetic resonance imaging and potential implications for neuro axial anesthesia. Rev Esp Anestesiol Reanim 2007;54:17383.

16. Reina MA, Villanueva MC, Lopez A, De Andres JA. Fat inside the dural sheath of lumbar nerve roots in humans. Rev Esp Anestesiol Reanim 2007;54:297301.

17. Bryant MS, Bremer AM, Nguyen TQ. Autogeneic fat 
transplants in the epidural space in routine lumbar spine surgery. Neurosurgery 1983;13:367-70.

18. Mohi Eldin MM, Abdel Razek NM. Epidural fibrosis after lumbar disc surgery: prevention and outcome evaluation. Asian Spine J 2015;9:370-85.

19. Yaman ME, Ergul G, Guvenc Y, et al. Evaluation of topical Dexmedetomidine administration in postlaminectomy epidural fibrosis rat model. Int J Surg 2018;53:80-5.

20. Fujita N, Ishihara S, Michikawa T, et al. Negative impact of spinal epidural lipomatosis on the surgical outcome of posterior lumbar spinous-splitting decompression surgery: a multicenter retrospective study. Spine J 2019;19:1977-85.

21. Sobti S, Grover A, John BP, Grewal SS, George UB. Prospective randomized comparative study to evaluate epidural fibrosis and surgical outcome in patients undergoing lumbar laminectomy with epidural autologous free fat graft or gelfoam: a preliminary study. Int J Appl Basic Med Res 2018;8:71-5.

22. Xu J, Chen Y, Yue Y, Sun J, Cui L. Reconstruction of epidural fat with engineered adipose tissue from adipose derived stem cells and PLGA in the rabbit dorsal laminectomy model. Biomaterials 2012;33:696573.

23. Abe T, Miyazaki M, Ishihara T, et al. Spinal epidural lipomatosis is associated with liver fat deposition and dysfunction. Clin Neurol Neurosurg 2019;185:105480.
24. Berthelot JM, Laredo JD, Darrieutort-Laffite C, Maugars Y. Stretching of roots contributes to the pathophysiology of radiculopathies. Joint Bone Spine 2018;85:41-5.

25. Boezaart AP, Prats-Galino A, Nin OC, et al. The posterior lumbar epidural space: three-dimensional reconstruction of high-resolution MRI: real and potential epidural spaces and their content in vivo. Pain Med 2019;20:1687-96.

26. Li C, Wang H, Liu H, Yin J, Cui L, Chen Z. The prevention effect of poly (L-glutamic acid)/chitosan on spinal epidural fibrosis and peridural adhesion in the post-laminectomy rabbit model. Eur Spine J 2014;23:2423-31.

27. Megan Sions J, Angelica Rodriguez C, Todd Pohlig R, Evan Hicks G, Charles Coyle P. Epidural fat and its association with pain, physical function, and disability among older adults with low back pain and controls. Pain Med 2018;19:1944-51.

28. Lee GW, Mun JU, Ahn MW. The impact of posterior epidural adipose tissue on postoperative outcomes after posterior decompression surgery for lumbar spinal stenosis: a prospectively randomized non-inferiority trial. J Orthop Surg (Hong Kong) 2020;28:2309499019896871.

29. Sung SE, Kang KK, Choi JH, et al. Comparisons of extracellular vesicles from human epidural fatderived mesenchymal stem cells and fibroblast cells. Int J Mol Sci 2021;22:2889. 